\title{
Sु \\ Spin-orbit coupling and broken spin degeneracy in multilayer graphene
}

\author{
Edward McCann ${ }^{1}$ and Mikito Koshino ${ }^{2}$ \\ ${ }^{1}$ Department of Physics, Lancaster University, Lancaster LA1 4YB, United Kingdom \\ ${ }^{2}$ Department of Physics, Tokyo Institute of Technology, 2-12-1 Ookayama, Meguro-ku, Tokyo 152-8551, Japan
}

(Received 30 April 2010; revised manuscript received 31 May 2010; published 15 June 2010)

\begin{abstract}
Since the lattices of $A B A$-stacked graphene multilayers with an even number of layers, as well as that of monolayer graphene, satisfy spatial-inversion symmetry, their electronic bands must be spin degenerate in the presence of time-inversion symmetry. In intrinsic monolayer and bilayer graphene, when symmetry is not broken by external fields, the only spin-orbit coupling present at low energy near the corner of the Brillouin zone is the Kane-Mele term, that opens a bulk energy gap but does not break the spin degeneracy of the energy bands [C. L. Kane and E. J. Mele, Phys. Rev. Lett. 95, 226801 (2005)]. However, spin splitting is allowed in multilayers with an odd number of layers $(\geq 3)$ because their lattices do not satisfy spatial-inversion symmetry. We show that, in trilayer graphene, in addition to the Kane-Mele term, there is a second type of intrinsic spin-orbit coupling present at low energy near the corner of the Brillouin zone. It introduces a Zeeman-type spin splitting of the energy bands at each valley with an opposite sign of the effective magnetic field in the two valleys. We estimate the magnitude of the effective field to be $\sim 2 \mathrm{~T}$.
\end{abstract}

DOI: 10.1103/PhysRevB.81.241409

PACS number(s): 73.22.Pr, 81.05.ue, 73.43.Cd

Interest in graphene ${ }^{1,2}$ stems partly from the presence of two Dirac cones in the low-energy electronic band structure, each of them supporting chiral quasiparticles. The origin of the Dirac-type spectrum lies in the fact that the honeycomb lattice of graphene has two inequivalent atomic sites, called $A$ and $B$, and the amplitude of the electronic wave function on them acts as an additional degree of freedom known as pseudospin. An "up" component of pseudospin pointing perpendicular to the graphene plane would correspond to electronic density solely on the $A$ sublattice sites, whereas "down" pseudospin corresponds to density on the $B$ sublattice. In practice, electronic density is usually shared equally between the sublattices so that the pseudospin is a linear combination of up and down, and it lies in the plane of the graphene sheet. There are two Dirac cones, centered at inequivalent corners of the Brillouin zone which are denoted $K_{+}$and $K_{-}$and are also referred to as valleys. The valleys introduce another spinlike degree of freedom into the electronic Hamiltonian.

The band structure described above is modified when the spin of the electron is taken into account. Kane and $\mathrm{Mele}^{3}$ introduced a spin-orbit coupling term that exists at the center of each valley,

$$
\mathcal{H}_{K M}=\alpha \Pi_{z} \sigma_{z} S_{z}
$$

where $\alpha$ is a parameter and Pauli matrices $\Pi_{z}, \sigma_{z}, S_{z}$ act in $K_{+} / K_{-}$valley, $A / B$ sublattice, and $\uparrow / \downarrow$ spin space, respectively. It satisfies time- and spatial-inversion symmetry requirements by coupling the out-of-plane component of electronic spin with the out-of-plane component of pseudospin. This does not break the spin and valley degeneracy of the energy bands, but it changes the balance between the $A$ and $B$ sublattices, opening a band gap and realizing a new, topological state of matter, a quantum spin-Hall insulator. ${ }^{3}$

In this Rapid Communication, we explain how time- and spatial-inversion symmetry influence spin and valley degeneracy in $A B A$-stacked (Bernal) multilayer graphene com- posed of $N$ layers. In the presence of time-reversal symmetry, when the atomic lattice satisfies spatial-inversion symmetry, and such symmetry is not broken by external fields, the electronic bands must be spin degenerate. This holds for monolayer and bilayer graphene where the only intrinsic spin-orbit coupling term ${ }^{3-8}$ present at low-energy near the center of the valley is of the Kane-Mele type, Eq. (1). However, the lattices of multilayers with odd $N(N \geq 3)$ do not satisfy spatialinversion symmetry so that spin splitting of the energy bands is allowed. We show that, in trilayer graphene, in addition to the Kane-Mele term, Eq. (1), there is a second type of intrinsic spin-orbit coupling present at low energy near the center of the valley,

$$
\mathcal{H}_{A B A}=\beta \Pi_{z} S_{z}
$$

It couples the out-of-plane component of electronic spin with the out-of-plane component of the valley "spin" degree of freedom. This introduces a Zeeman-type spin splitting of the energy bands at each valley, of magnitude proportional to the parameter $\beta$, with an opposite sign of the effective Zeeman field in the two valleys.

We begin by briefly describing how time and spatial symmetries impose constraints on the spectra of graphene multilayers, before discussing the particular examples of bilayer and trilayer graphene. We consider intrinsic spin-orbit terms that exist in samples in the absence of external electric or magnetic fields. For example, we neglect the influence of a transverse electric field that breaks reflection symmetry and produces an additional, Rashba-type term. ${ }^{3-5,9}$ Time-reversal symmetry relates energy bands with opposite spin components $\{\uparrow, \downarrow\}$ and momenta $\mathbf{k}$ and $-\mathbf{k}$ : $\boldsymbol{\epsilon}_{\uparrow}(\mathbf{k})=\boldsymbol{\epsilon}_{\downarrow}(-\mathbf{k})$. In monolayer graphene, ${ }^{3}$ and in multilayers with even $N$, including bilayers, ${ }^{10-13}$ the lattice, Figs. 1(a) and 1(b), obeys spatialinversion symmetry $(x, y, z) \rightarrow(-x,-y,-z)$, which relates energy bands with the same spin component (as spin is an axial vector) and opposite momenta: $\epsilon_{\uparrow}(\mathbf{k})=\epsilon_{\uparrow}(-\mathbf{k})$. Combining time- and spatial-inversion symmetry guarantees spin degen- 


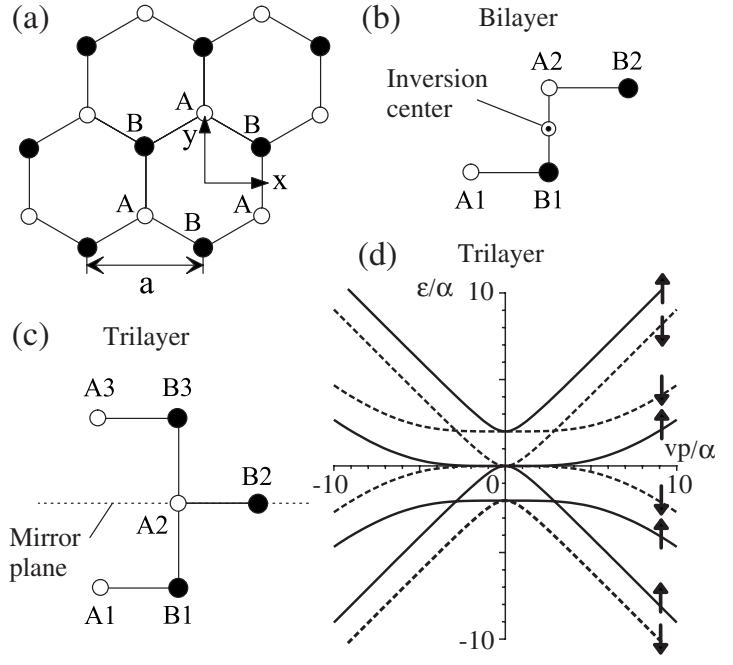

FIG. 1. (a) Plan view of the lattice of a single layer of graphene showing inequivalent $A$ and $B$ sites. (b) Schematic side view of the unit cell of bilayer graphene and (c) of Bernal-stacked trilayer graphene, showing the inversion center for bilayer and the mirror plane for trilayer. (d) Sketch of the trilayer energy bands in the vicinity of the valley center, using Eqs. (7) and (8), where we use $a_{m}=a_{b}=b_{m}=b_{b}=0, \alpha_{m}=\alpha_{b}=\beta_{m}=-\beta_{b} \equiv \alpha$ with the value $\alpha=\gamma_{1} / 20$ chosen for illustrative purposes.

eracy of the energy bands, $\boldsymbol{\epsilon}_{\uparrow}(\mathbf{k})=\boldsymbol{\epsilon}_{\downarrow}(\mathbf{k})$, even in the presence of finite spin-orbit coupling. The situation is different in multilayers with odd $N$, starting from trilayer graphene $(N=3)$, Fig. 1(c), because their lattices do not satisfy spatialinversion symmetry. ${ }^{14-16}$ When time-reversal symmetry holds, the energy of bands with opposite spin and momenta must still be equal, $\epsilon_{\uparrow}(\mathbf{k})=\epsilon_{\downarrow}(-\mathbf{k})$, but spin splitting of the energy bands at the same momentum, $\boldsymbol{\epsilon}_{\uparrow}(\mathbf{k}) \neq \boldsymbol{\epsilon}_{\downarrow}(\mathbf{k})$, is allowed, Fig. 1(d).

Bilayer graphene. In multilayers with an even number of layers, the lattice is symmetric with respect to spatialinversion symmetry $P[(x, y, z) \rightarrow(-x,-y,-z)]$, because the point group of the lattice $D_{3 d}$ (Refs. 14 and 15) $\left(\left\{E, 2 C_{3}, 3 C_{2}^{\prime}, i, 2 S_{6}, 3 \sigma_{d}\right\}\right)$ can be regarded as a direct product of group $D_{3}\left(\left\{E, 2 C_{3}, 3 C_{2}^{\prime}\right\}\right)$ with the inversion group $C_{i}(\{E, i\})$. Here, we focus on the particular case of bilayer graphene, ${ }^{10-13}$ which consists of two coupled layers of carbon atoms arranged on a honeycomb lattice with inequivalent sites $\{A 1, B 1\}$ and $\{A 2, B 2\}$ on the bottom and top layers, respectively, Fig. 1(b). The low-energy tight-binding model takes into account a single $p_{z}$ orbital per site, with parameter $\gamma_{0}$ taking into account nearest-neighbor hopping, $A 1-B 1$ and $A 2-B 2$, within each layer. The layers are arranged so that sites $B 1$ and $A 2$ are directly below and above each other, connected by interlayer coupling $\gamma_{1} \sim 0.4 \mathrm{eV}$. Then, the band structure consists of four bands. Two of them, corresponding to the coupled orbitals on the $B 1$ and $A 2$ sites, are split away from zero energy by $\pm \gamma_{1}$. The other two bands, formed by effective hopping between $A 1$ and $B 2$ sites, are parabolic and touching near zero energy.

The parabolic low-energy bands may be described using a basis of electronic wave functions with components $\psi_{i, \xi, s}$, where $i=\{A 1, B 2\}$ labels the relevant lattice sites, $s=\{\uparrow, \downarrow\}$ spin components, and $\xi= \pm 1$ distinguishes between the valleys located at $\mathbf{K}_{\xi}=\xi\left(\frac{4}{3} \pi a^{-1}, 0\right)$ with lattice constant $a$. Neglecting spin, the low-energy bands are approximately described by the effective Hamiltonian ${ }^{11} \mathcal{H}_{2}^{(0)}=$ $-\left(v^{2} / \gamma_{1}\right) S_{0}\left(\Pi_{0} \sigma_{x}\left[p_{x}^{2}-p_{y}^{2}\right]+2 \Pi_{z} \sigma_{y} p_{x} p_{y}\right)$, where we use direct products of Pauli matrices $\sigma_{x, y, z}, \sigma_{0} \equiv \hat{1}$ acting in the $A 1 / B 2$ sublattice space, $\Pi_{x, y, z}, \Pi_{0} \equiv \hat{1}$ acting in the valley space, and $S_{x, y, z}, S_{0} \equiv \hat{1}$ acting in the spin space. Here, $v=(\sqrt{3} / 2) a \gamma_{0} / \hbar$ is the monolayer Fermi velocity and $\mathbf{p}=\left(p_{x}, p_{y}\right)$ is the momentum measured with respect to the center of the valley, $\mathbf{k}=\hbar \mathbf{K}_{\xi}+\mathbf{p}$.

We begin by exploring which spin-orbit terms, if any, satisfy time and space symmetries of the lattice. Apart from symmetry, we assume that low-energy states are composed from orbitals on the $A 1, B 2$ sites so that the following argument does not rely on details of a particular model. In the above basis, the time-reversal operator is $T=\Pi_{x} \sigma_{0} S_{y} \mathcal{K}$, where $\mathcal{K}$ represents complex conjugation, and translation by a distance equal to the lattice constant $a$ along the $x$ axis is given by $e^{i 4 \pi \Pi_{z} / 3}$. Generators of the point group of the lattice $D_{3 d}$ are an active rotation anticlockwise by angle $2 \pi / 3, C_{3}$ $=-e^{i \pi \Pi_{z} \sigma_{z} / 3} e^{-i \pi S_{z} / 3}$, rotation by angle $\pi$ about the $x$ axis, $C_{2}^{\prime}$ $=-i \Pi_{0} \sigma_{x} S_{x}$, and spatial inversion, $i=\Pi_{x} \sigma_{x} S_{0}$. We focus on the center of the valley, $p=|\mathbf{p}|=0$, and consider all possible terms describing coupling between valley, sublattice, and spin of the form $\Pi_{i} \sigma_{j} S_{k}$, where $i=\{0, x, y, z\}, j=\{0, x, y, z\}$, and $k=\{0, x, y, z\}$. Then, we determine which of them is invariant with respect to the time and space symmetries of the bilayer lattice. Except for a constant term $\Pi_{0} \sigma_{0} S_{0}$, there is only one, which is a term of the Kane-Mele type, Eq. (1). ${ }^{3-8}$ As in a monolayer, ${ }^{3}$ it leads to the opening of a gap at the center of the valley, but does not break spin and valley degeneracy.

Trilayer graphene. In odd- $N$ multilayers with $N \geq 3$, the point group $D_{3 h}\left(\left\{E, 2 C_{3}, 3 C_{2}^{\prime}, \sigma_{h}, 2 S_{3}, 3 \sigma_{v}\right\}\right)$ (Refs. 14 and 15) can be regarded as a direct product of the group $D_{3}\left(\left\{E, 2 C_{3}, 3 C_{2}^{\prime}\right\}\right)$ with the reflection group $C_{s}\left(\left\{E, \sigma_{h}\right\}\right)$. With respect to the even- $N$ multilayers, spatial inversion is replaced by mirror reflection $\sigma_{h}[(x, y, z) \rightarrow(x, y,-z)]$. Trilayer graphene $\mathrm{e}^{13,14,16-22}$ consists of three coupled layers of carbon atoms arranged on a honeycomb lattice with inequivalent sites $\{A 1, B 1\},\{A 2, B 2\}$, and $\{A 3, B 3\}$ on the bottom, middle, and top layers, respectively, Fig. 1(c). The simple tight-binding model using a single $p_{z}$ orbital per atom includes coupling $\gamma_{0}$ between nearest-neighbor sites within each layer, $A 1-B 1, A 2-B 2$, and $A 3-B 3$, as well as interlayer coupling $\gamma_{1}$ between sites $B 1$ and $A 2$, and $A 2$ and $B 3$, that are directly below and above each other.

Linear combinations of atomic orbitals may be separated $^{16,18}$ into a pair that are odd with respect to mirror reflection symmetry $\sigma_{h}, \phi_{1, \xi, s}^{(m)}=\left[\psi_{A 1, \xi, s}-\psi_{A 3, \xi, s}\right] / \sqrt{2}, \phi_{2, \xi, s}^{(m)}$ $=\left[\psi_{B 1, \xi, s}-\psi_{B 3, \xi, s}\right] / \sqrt{2}$, and four that are even with respect to $\sigma_{h}, \phi_{1, \xi, s}^{(b)}=\left[\psi_{A 1, \xi, s}+\psi_{A 3, \xi, s}\right] / \sqrt{2}, \quad \phi_{2, \xi, s}^{(b)}=\psi_{B 2, \xi, s}, \quad \phi_{3, \xi, s}^{(b)}=\psi_{A 2, \xi, s}$, and $\phi_{4, \xi, s}^{(b)}=\left[\psi_{B 1, \xi, s}+\psi_{B 3, \xi, s}\right] / \sqrt{2}$. Written in a basis of states $\phi_{i, \xi, s}^{(m / b)}$, the Hamiltonian of trilayer graphene separates into two parts. ${ }^{16,18}$ The first has a basis of odd orbitals $\phi_{1, \xi, s}^{(m)}$, $\phi_{2, \xi, s}^{(m)}$, and it has a form analogous to that of monolayers, 


$$
\mathcal{H}_{m}^{(0)}=v S_{0}\left(\Pi_{z} \sigma_{x}^{(m)} p_{x}+\Pi_{0} \sigma_{y}^{(m)} p_{y}\right),
$$

where matrices $\sigma_{0, x, y, z}^{(m)}$ act in the $\phi_{1}^{(m)}, \phi_{2}^{(m)}$ sublattice space. The second part of the Hamiltonian has a basis of four even orbitals $\phi_{i, \xi, s}^{(b)}$ resulting in four parabolic bilayerlike bands. As in a bilayer, two bands touch near zero energy. They are related to orbitals $\phi_{1, \xi, s}^{(b)}, \phi_{2, \xi, s}^{(b)}$ and may be described by an effective quadratic Hamiltonian,

$$
\mathcal{H}_{b}^{(0)}=-\frac{v^{2} S_{0}}{\sqrt{2} \gamma_{1}}\left[\Pi_{0} \sigma_{x}^{(b)}\left(p_{x}^{2}-p_{y}^{2}\right)+2 \Pi_{z} \sigma_{y}^{(b)} p_{x} p_{y}\right],
$$

where matrices $\sigma_{0, x, y, z}^{(b)}$ act in the $\phi_{1}^{(b)}, \phi_{2}^{(b)}$ space.

An analysis of the transformation properties of the trilayer Hamiltonian under symmetries of the lattice is relatively simple in the basis of orbitals $\phi_{i, \xi, s}^{(m / b)}$ because time and spatial symmetry operators do not mix the monolayerlike and bilayerlike parts of the Hamiltonian. We therefore consider them separately. In the monolayerlike part, the time-reversal operator is $T=\Pi_{x} \sigma_{0}^{(m)} S_{y} \mathcal{K}$ and translation by a distance equal to the lattice constant $a$ along the $x$ axis is given by $e^{i 4 \pi \Pi_{z} / 3}$. Generators of the group $D_{3 h}$ are an active rotation anticlockwise by angle $2 \pi / 3, C_{3}=e^{-i \pi S_{z} / 3}\left[\left(1-\sigma_{z}^{(m)}\right) / 2+e^{-i 2 \pi \Pi_{z} / 3}(1\right.$ $\left.\left.+\sigma_{z}^{(m)}\right) / 2\right]$, rotation by angle $\pi$ about the $y$ axis, $C_{2}^{\prime}$ $=i \Pi_{x} \sigma_{0}^{(m)} S_{y}$, and mirror reflection symmetry, $\sigma_{h}=i \Pi_{0} \sigma_{0}^{(m)} S_{z}$.

We focus on the center of the valley, $p=0$, and consider all possible terms describing coupling between valley, sublattice, and spin of the form $\Pi_{i} \sigma_{j} S_{k}$, where $i=\{0, x, y, z\}, j$ $=\{0, x, y, z\}$, and $k=\{0, x, y, z\}$. Then, we determine which of them is invariant with respect to the time and space symmetries of the lattice,

$$
\begin{aligned}
\mathcal{H}_{m}^{(1)}= & a_{m} \Pi_{0} \sigma_{0}^{(m)} S_{0}+b_{m} \Pi_{0} \sigma_{z}^{(m)} S_{0}+\alpha_{m} \Pi_{z} \sigma_{z}^{(m)} S_{z} \\
& +\beta_{m} \Pi_{z} \sigma_{0}^{(m)} S_{z} .
\end{aligned}
$$

The first two terms of Eq. (5) are spin independent and the second two are spin-orbit terms. We repeat the symmetry analysis for the bilayerlike part of the Hamiltonian. We consider the two low-energy bands that touch near zero energy, related to orbitals $\phi_{1, \xi, s}^{(b)}, \phi_{2, \xi, s}^{(b)}$. For that basis, $T=\Pi_{x} \sigma_{0}^{(b)} S_{y} \mathcal{K}$, translation is $e^{i 4 \pi \Pi_{z} / 3}, C_{3}=e^{-i \pi S_{z} / 3} e^{-i 2 \pi \Pi_{z} \sigma_{z}^{(b)} / 3}, C_{2}^{\prime}=i \Pi_{x} \sigma_{z}^{(b)} S_{y}$, and $\sigma_{h}=-i \Pi_{0} \sigma_{0}^{(b)} S_{z}$. The invariant terms at the center of the valley are

$$
\mathcal{H}_{b}^{(1)}=a_{b} \Pi_{0} \sigma_{0}^{(b)} S_{0}+b_{b} \Pi_{0} \sigma_{z}^{(b)} S_{0}+\alpha_{b} \Pi_{z} \sigma_{z}^{(b)} S_{z}+\beta_{b} \Pi_{z} \sigma_{0}^{(b)} S_{z}
$$

and they are analogous to the terms in the monolayerlike part, Eq. (5).

The monolayerlike $\left[\mathcal{H}_{m}^{(0)}+\mathcal{H}_{m}^{(1)}\right]$ and bilayerlike $\left[\mathcal{H}_{b}^{(0)}\right.$ $\left.+\mathcal{H}_{b}^{(1)}\right]$ parts of the Hamiltonian produce superimposed monolayerlike $\epsilon_{m}$ and bilayerlike $\epsilon_{b}$ bands,

$$
\begin{gathered}
\epsilon_{m}=a_{m}+s \xi \beta_{m} \pm \sqrt{\left(b_{m}+s \xi \alpha_{m}\right)^{2}+v^{2} p^{2}}, \\
\epsilon_{b}=a_{b}+s \xi \beta_{b} \pm \sqrt{\left(b_{b}+s \xi \alpha_{b}\right)^{2}+v^{4} p^{4} /\left(2 \gamma_{1}^{2}\right)},
\end{gathered}
$$

where $s= \pm 1$ denotes different spin components, $\xi= \pm 1$ different valleys, and we consider $\left\{\left|\epsilon_{m / b}\right|, v p\right\} \ll \gamma_{1}$. Figure $1(\mathrm{~d})$ shows a plot of the trilayer energy bands, using Eqs. (7) and
(8), where we use $a_{m}=a_{b}=b_{m}=b_{b}=0, \alpha_{m}=\alpha_{b}=\beta_{m}=-\beta_{b} \equiv \alpha$ and the value $\alpha=\gamma_{1} / 20$.

The terms proportional to the parameters $a_{m}$ and $a_{b}$ in Eqs. (5) and (6) produce constant energy shifts of the monolayerlike and bilayerlike spectra with respect to each other. The term proportional to $b_{m}\left(b_{b}\right)$ is absent in a monolayer (bilayer) but allowed in trilayers owing to the absence of spatial-inversion symmetry. It breaks the $\phi_{1}^{(m)} / \phi_{2}^{(m)}$ $\left(\phi_{1}^{(b)} / \phi_{2}^{(b)}\right)$ sublattice symmetry and opens a gap between the monolayer (bilayer) bands but does not generally break valley symmetry. ${ }^{16}$ The Kane-Mele spin-orbit term, responsible for factors $\alpha_{m / b}$, has been discussed in detail elsewhere. ${ }^{3-8}$ Here, we note that, in trilayers, it can conspire with the $b_{m / b}$ parameters to break spin degeneracy even at zero magnetic field. Parameters $\beta_{m / b}$ arise from the spin-orbit term unique to odd- $N$ multilayers and they describe a Zeeman-type spin splitting of the energy bands at each valley with an opposite sign of the effective Zeeman field in the two valleys.

The Hamiltonians, Eqs. (5) and (6), were obtained using symmetry arguments but it is possible to relate the phenomenological parameters to tight-binding parameters. For the spin-independent part of the Hamiltonian, parameters $a_{m}, b_{m}$, $a_{b}$, and $b_{b}$ may be related to next-nearest-layer coupling parameters $\gamma_{2}$ (between $A 1$ and $A 3$ ) and $\gamma_{5}$ (between $B 1$ and B3) as ${ }^{16} a_{m}=-\left(\gamma_{2}+\gamma_{5}\right) / 2, b_{m}=-\left(\gamma_{2}-\gamma_{5}\right) / 2, a_{b}=\gamma_{2} / 2$, and $b_{b}=\gamma_{2} / 2$.

While it is generally accepted that the magnitude $\alpha$ of the intrinsic spin-orbit coupling near the center of the valleys in graphene is small, ${ }^{3,23}$ its value has been the subject of theoretical debate. ${ }^{4,5,7,8,24-26}$ It was recently proposed ${ }^{26}$ that coupling between the $p_{z}$ orbitals and $d$ orbitals gives the dominant contribution to spin-orbit coupling in monolayers, because $p_{z}$ orbitals are not orthogonal to $d_{x z}$ and $d_{y z}$ orbitals, yielding $\alpha \sim 0.01 \mathrm{meV}$. Very recently, it has been suggested ${ }^{7,8}$ that spin-orbit coupling in bilayer graphene can be relatively large due to coupling between $p_{z}$ orbitals and $\sigma$ bands on different layers (that are not orthogonal), giving a contribution to the spin-orbit coupling $\alpha \sim 0.1 \mathrm{meV}$.

Using the tight-binding model, we generalize the estimate of the magnitude of spin-orbit coupling in monolayers ${ }^{4,5,24}$ and bilayers ${ }^{7,8}$ to demonstrate that both types of intrinsic spin-orbit coupling are likely to be as large in trilayers as the Kane-Mele term in bilayers: $\left|\beta_{m / b}\right| \sim\left|\alpha_{m / b}\right| \sim 0.1 \mathrm{meV}$. We take into account coupling of the $p_{z}$ and $\sigma$ orbitals, by writing the tight-binding Hamiltonian including the Hamiltonian of the $p_{z}$ orbitals $H_{\pi}$ and of the $\sigma$ orbitals $H_{\sigma}$ as

$$
H=\left(\begin{array}{cc}
H_{\pi} & V \\
V^{\dagger} & H_{\sigma}
\end{array}\right)
$$

where matrix $V$ describes coupling between the $p_{z}$ and $\sigma$ orbitals. Here, $H_{\pi}$ is a $12 \times 12$ matrix (describing a spin-up and spin-down $p_{z}$ orbital per site, and six sites in the trilayer unit cell) and $H_{\sigma}$ is a $36 \times 36$ matrix (six orbitals per site taking into account two spins and $s, p_{x}$ and $p_{y}$, and six sites in the trilayer unit cell).

Block $H_{\sigma}$ contains interatomic matrix elements that are written using Slater and Koster matrix elements. ${ }^{27}$ For example, at the valley center, the matrix element between a $p_{x}$ 
orbital on an $A j$ site and a $p_{y}$ orbital on an adjacent $B j$ site within the same layer $(j=1,2,3)$ is $\left\langle p_{x}^{A j}|H| p_{y}^{B j}\right\rangle=-3 i \xi V_{p p} / 4$. Matrix $V$ in Eq. (9) describes the coupling between $p_{z}$ orbitals and $\sigma$ orbitals. We take into account skew interlayer coupling between orbitals on sites $A 1, B 2$, and $A 3$, that are not coupled by $\gamma_{1}$ (such coupling, if it is only between $p_{z}$ orbitals, is usually denoted $\gamma_{3}$ ). For example, the matrix elements between a $p_{z}$ orbital on the $A 1$ site and a $p_{x}$ or $p_{y}$ orbital on the $B 2$ site are $\left\langle p_{z}^{A 1}|H| p_{x}^{B 2}\right\rangle=3 i \xi V_{p p}^{\prime} / 2$ and $\left\langle p_{z}^{A 1}|H| p_{y}^{B 2}\right\rangle=$ $-3 V_{p p}^{\prime} / 2$ exactly at the valley center. For an $A 3$ site instead of $A 1$, these matrix elements acquire an additional minus sign.

For the spin-orbit interaction, we include intra-atomic coupling $H=\lambda_{0} \mathbf{L} \cdot \mathbf{S}$ between the $p$ orbitals. This introduces spin-dependent matrix elements within $H_{\sigma}$ for the same atomic site $j$ such as $\left\langle p_{x, \uparrow}^{j}|H| p_{y, \uparrow}^{j}\right\rangle=-i \lambda_{0}$ and $\left\langle p_{x, \downarrow}^{j}|H| p_{y, \downarrow}^{j}\right\rangle$ $=i \lambda_{0}$. The effective Hamiltonian describing the $p_{z}$ orbitals at zero energy may be written using second-order perturbation theory as $H_{\mathrm{eff}} \approx H_{\pi}-V H_{\sigma}^{-1} V^{\dagger}$. Doing so, we recover the intrinsic spin-orbit terms, Eqs. (5) and (6), where $\alpha_{m}=\alpha_{b}$ $=\beta_{m}=-\beta_{b}=2 \lambda_{0}\left(V_{p p}^{\prime} / V_{p p}\right)^{2}$. The magnitude of this estimate is the same as that for the Kane-Mele term in bilayer graphene. ${ }^{7}$ Using $V_{p p}^{\prime} / V_{p p} \sim 0.1$ and $\lambda_{0} \sim 10 \mathrm{meV}$ yields $\left|\alpha_{m / b}\right|=\left|\beta_{m / b}\right| \sim 0.1 \mathrm{meV}$. We compare the spin splitting of the bands, $2\left|\beta_{m / b}\right|$, to the Zeeman energy $g \mu_{B} B$ in a real external field $B$. Using $g=2$ gives an effective field of $B$ $\sim 2 \mathrm{~T}$, which is comparable to the real fields required to observe Zeeman-split conductance fluctuations in graphene. ${ }^{28}$ Note that other techniques sensitive to spin-split energy bands include optical spectroscopy ${ }^{29}$ and spinresolved photoemission. ${ }^{30}$

The authors thank V. I. Fal'ko and H. Schomerus for discussions. This project has been funded by EPSRC First Grant No. EP/E063519/1 and by JST-EPSRC Japan-UK Cooperative Programme under Grant No. EP/H025804/1.
${ }^{1}$ K. S. Novoselov et al., Science 306, 666 (2004).

${ }^{2}$ A. H. Castro Neto et al., Rev. Mod. Phys. 81, 109 (2009).

${ }^{3}$ C. L. Kane and E. J. Mele, Phys. Rev. Lett. 95, 226801 (2005).

${ }^{4}$ H. Min, J. E. Hill, N. A. Sinitsyn, B. R. Sahu, L. Kleinman, and A. H. MacDonald, Phys. Rev. B 74, 165310 (2006).

${ }^{5}$ D. Huertas-Hernando, F. Guinea, and A. Brataas, Phys. Rev. B 74, 155426 (2006).

${ }^{6}$ R. van Gelderen and C. Morais Smith, Phys. Rev. B 81, 125435 (2010).

${ }^{7}$ F. Guinea, arXiv:1003.1618 (unpublished).

${ }^{8}$ H. Liu, X. Xie, and Q. Sun, arXiv:1004.0881 (unpublished).

${ }^{9}$ Y. A. Bychkov and E. I. Rashba, J. Phys. C 17, 6039 (1984).

${ }^{10}$ K. S. Novoselov et al., Nat. Phys. 2, 177 (2006).

${ }^{11}$ E. McCann and V. I. Fal'ko, Phys. Rev. Lett. 96, 086805 (2006).

${ }^{12}$ T. Ohta et al., Science 313, 951 (2006).

${ }^{13}$ F. Guinea, A. H. Castro Neto, and N. M. R. Peres, Phys. Rev. B 73, 245426 (2006).

${ }^{14}$ S. Latil and L. Henrard, Phys. Rev. Lett. 97, 036803 (2006).

${ }^{15}$ J. L. Mañes, F. Guinea, and M. A. H. Vozmediano, Phys. Rev. B 75, 155424 (2007).

${ }^{16}$ M. Koshino and E. McCann, Phys. Rev. B 79, 125443 (2009); 81, 115315 (2010).

${ }^{17}$ B. Partoens and F. M. Peeters, Phys. Rev. B 74, 075404 (2006); 75, 193402 (2007).

${ }^{18}$ M. Koshino and T. Ando, Phys. Rev. B 76, 085425 (2007); 77,
115313 (2008); Solid State Commun. 149, 1123 (2009).

${ }^{19}$ C. L. Lu, C. P. Chang, Y. C. Huang, R. B. Chen, and M. L. Lin, Phys. Rev. B 73, 144427 (2006).

${ }^{20}$ M. F. Craciun et al., Nat. Nanotechnol. 4, 383 (2009).

${ }^{21}$ M. Aoki and H. Amawashi, Solid State Commun. 142, 123 (2007).

${ }^{22}$ A. A. Avetisyan, B. Partoens, and F. M. Peeters, Phys. Rev. B 79, 035421 (2009); 80, 195401 (2009).

${ }^{23}$ G. Dresselhaus and M. S. Dresselhaus, Phys. Rev. 140, A401 (1965).

${ }^{24}$ Y. Yao, F. Ye, X. L. Qi, S. C. Zhang, and Z. Fang, Phys. Rev. B 75, 041401(R) (2007).

${ }^{25}$ J. C. Boettger and S. B. Trickey, Phys. Rev. B 75, 121402(R) (2007).

${ }^{26}$ M. Gmitra, S. Konschuh, C. Ertler, C. Ambrosch-Draxl, and J. Fabian, Phys. Rev. B 80, 235431 (2009).

${ }^{27}$ J. C. Slater and G. F. Koster, Phys. Rev. 94, 1498 (1954).

${ }^{28}$ M. B. Lundeberg and J. A. Folk, Nat. Phys. 5, 894 (2009).

${ }^{29}$ D. Gammon, A. L. Efros, T. A. Kennedy, M. Rosen, D. S. Katzer, D. Park, S. W. Brown, V. L. Korenev, and I. A. Merkulov, Phys. Rev. Lett. 86, 5176 (2001).

${ }^{30}$ Yu. S. Dedkov, M. Fonin, U. Rüdiger, and C. Laubschat, Phys. Rev. Lett. 100, 107602 (2008); A. Varykhalov, J. SanchezBarriga, A. M. Shikin, C. Biswas, E. Vescovo, A. Rybkin, D. Marchenko, and O. Rader, ibid. 101, 157601 (2008). 